\title{
Chronische Erschöpfung statt Neuroborreliose
}

\author{
Patienten mit der Diagnose "Post-Lyme-Syndrom" \\ ähneln solchen mit chronischer Fatigue, vor allem \\ wenn eine Borreliose nicht mit validierten Labor- \\ standards, sondern mit „alternativen Tests" diagnos- \\ tiziert worden ist. Sie profitieren auch nicht von einer \\ Langzeitantibiose. Patienten mit Post-Lyme-Syndrom \\ haben wohl eher ein psychisches Problem.
}

Patienten mit Neuroborreliose lassen sich eigentlich sehr gut behandeln, nach aktuellen Untersuchungen sprechen die allermeisten auf eine Antibiose an. Daran erinnerte Professor Jörg Weber vom Klinikum in Klagenfurt. Auf der Fortbildungsveranstaltung Neuro Update in Mainz stellte er eine slowenische Untersuchung bei 77 Patienten mit Bannwarth-Syndrom vor [1]. Bei allen konnte eine Liquorpleozytose neben einer Radikuloneuritis nachgewiesen werden, alle unterzogen sich einer zweibis dreiwöchigen Therapie mit Ceftriaxon oder Doxycyclin. 14 Tage nach Beginn der Antibiose waren die Symptome bei $88 \%$ der Patienten fast oder komplett verschwunden. 20 Betroffene erhielten aufgrund einer anhaltenden Liquorpleozytose und/ oder Beschwerden eine erneute Antibiose, neun (12\%) hatten langfristig Beschwerden. Diese Patienten hatten zunächst kaum auf die Antibiose angesprochen.

Ein Problem scheint nach diesen Daten noch immer die recht späte Diagnose zu sein. So wurde eine Neuroborreliose im Schnitt erst 30 Tage nach Beginn der neurologischen Symptome diagnostiziert; immerhin waren periphere Fazialisparesen und ausgeprägte Lähmungen um die Hälfte bis vier Fünftel seltener zu beobachten als in früheren Untersuchungen.

\section{Kaum Unterschiede zum chronischen Fatigue-Syndrom}

Weber verwies auch auf eine US-Langzeitstudie mit 101 Patienten, die unterschiedliche Borreliose-Manifestationen aufwiesen. Nach drei Jahren wurden die Betroffenen mithilfe eines strukturierten Gesundheitsfragebogens (SF-36) untersucht. Dabei zeigten sich keinerlei Abweichungen zur Allgemeinbevölkerung.

In einer weiteren US-Untersuchung von Patienten mit per Kultur nachgewiesener Borreliose stellten die Ärzte nach 15 Jahren nur bei $5 \%$ ein Post-Lyme-Syndrom fest, bei keinem Betroffenen bestanden jedoch funktionelle Einschränkungen. Die Diagnose Post-Lyme-Syndrom sei daher kritisch zu hinterfragen, vor allem dann, wenn eine Borreliose nicht mit den gängigen Laborstandards, sondern mit „alternativen Tests“ diagnostiziert worden sei, sagte Weber. Dazu zählen etwa Antikörpernachweise per Western-Blot-Analyse, die bei mehr als der Hälfte der Getesteten ohne Borreliose falsch positive Resultate liefern. In einer kanadischen Untersuchung hatten Forscher 13 solcher Patienten mit alternativ diagnostizierter chronischer Lyme-Borreliose mit 25 Patienten verglichen, bei denen ein chronisches Fatigue-Syndrom (CFS) festgestellt worden war, sowie mit 25 gesunden Probanden und elf mit einem systemischen Lupus erythematodes (SLE) [2]. Alle füllten diverse Fragebögen zu Lebensqualität, funktionellen Einschränkungen, Fatigue, Schlafqualität und zum psychischen und allgemeinen

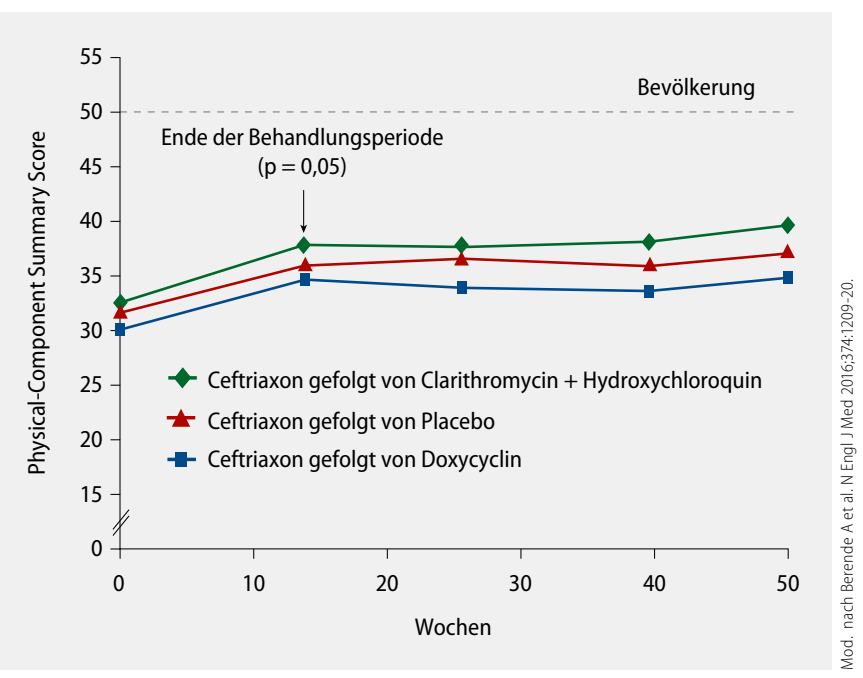

1 Langzeittherapie der Borreliose. Von 280 Patienten mit Post-Lyme-Syndrom ging es den Patienten der Antibiotika-Gruppen nicht besser als denen der Placebogruppe.

Gesundheitszustand aus. Dabei zeigten sich praktisch keine Unterschiede zwischen Patienten mit "alternativer Borreliose“ und solchen mit CFS. Beide Gruppen fühlten sich den Anforderungen einer Vollzeitbeschäftigung nicht gewachsen, klagten über Schlafprobleme und ausgeprägte Fatigue. Auch zeigten sie ähnlich schlechte Werte auf funktionellen Skalen und beim psychischen Zustand. Beide Gruppen unterschieden sich dabei deutlich von Gesunden und Patienten mit SLE. „Patienten mit Post-Lyme-Syndrom haben wohl eher ein psychisches Problem", so Weber.

\section{Zwölf-Wochen-Antibiose ohne Effekt}

Kein Wunder, dass ihnen auch eine verlängerte Antibiose nichts nützt. Darauf deutet jedenfalls eine niederländische Studie mit 280 Patienten mit Post-Lyme-Syndrom [3]. Bei ihnen war in der Vergangenheit eine Borreliose diagnostiziert worden oder es ließen sich Immunoblot-bestätigt IgG- und IgM-Antikörper auf Borrelien zeitgleich mit den Beschwerden nachweisen. Alle bekamen zunächst für zwei Wochen Ceftriaxon, ein Drittel erhielt anschließend zwölf Wochen lang Doxycyclin, ein weiteres Drittel Clarithromycin und Hydroxychloroquin, die übrigen Placebo ( Abb. 1). Nach drei Monaten fühlten sich die Patienten in den Antibiotika-Gruppen auch nicht besser als die mit Placebo. Ein Jahr später gab es ebenfalls keine Unterschiede.

Thomas Müller, Springer Medizin

Neuroinfektiologie (Prof. Jörg Weber, Klagenfurt). Neuro Update, Mainz, 3.3.2017

\footnotetext{
Literatur:

1. Ogrinc K et al. Clin Infect Dis 2016; 63 (3): 346-53

2. Patrick DM et al. Clin Infect Dis 2015; 61 (7): 1084-91

3. Berende A et al. N Engl J Med 2016; 374: 1209-20
} 